\title{
HYPERGROUPS ASSOCIATED TO HARMONIC NA GROUPS
}

\author{
BIANCA DI BLASIO
}

(Received 31 May 2000; revised 13 January 2001)

\author{
Communicated by A. H. Dooley
}

\begin{abstract}
A harmonic $N A$ group is a suitable solvable extension of a two-step nilpotent Lie group $N$ of Heisenberg type by $\mathbb{R}^{+}$, which acts on $N$ by anisotropic dilations. A hypergroup is a locally compact space for which the space of Borel measures has a convolution structure preserving the probability measures and satisfying suitable conditions. We describe a class of hypergroups associated to NA groups.
\end{abstract}

2000 Mathematics subject classification: primary 43A62, $43 \mathrm{A80}$.

Keywords and phrases: hypergroups, harmonic spaces.

\section{Introduction}

A hypergroup is a locally compact space $X$ for which the space $M(X)$ of Borel measures on $X$ is a $*$-algebra satisfying suitable conditions (see [11]). Since any symmetric space $S$ has a hypergroup $X(S)$ naturally associated to it, Wildberger [12] pointed out the following problem: for what more general Riemannian manifold $S$ does the hypergroup $X(S)$ make sense? In this paper we partially answer this question: we introduce new examples of hypergroups associated to a class of harmonic spaces. We recall that a Riemannian manifold is said to be harmonic if the volume density depends only on the geodesic distance from a fixed point.

The $N A$ groups considered in the paper are one-dimensional solvable extensions of two-step nilpotent Lie groups $N$ of Heisenberg type equipped with a suitable Riemannian metric [7]. The class of these $N A$ groups includes all noncompact symmetric spaces of rank one (the real hyperbolic spaces fit into this framework as degenerate cases); however most of them are nonsymmetric harmonic spaces [7]. So far this class of manifolds provides the only known examples of nonsymmetric harmonic spaces.

(C) 2002 Australian Mathematical Society $1446-7887 / 2002 \$ A 2.00+0.00$ 
In the paper we briefly describe hypergroups associated to $N A$ groups and their main properties. We give the explicit expression for the convolution structure, which can be deduced from a complicated formula proved by Flensted-Jensen and Koornwinder [9]. Finally we notice that hypergroups associated to $N A$ groups of the same dimension and such that the center of the Lie algebra $\mathfrak{n}$ of $N$ have the same dimension are all isomorphic.

\section{Notation}

Denote by $M(X)$ the space of Radon measures on a locally compact space $X$ and by $M_{1}(X)$ the subset of $M(X)$ of probability measures. The space $M(X)$ is the dual of $C_{0}(X)$, the space of continuous functions on $X$ which vanish at infinity. We shall write

$$
(f, \nu)=\int_{X} f(r) d \nu(r) \quad f \in C_{0}(X), \nu \in M(X) .
$$

\section{Hypergroups}

DEFINITION ([11]). A locally compact space $X$ is a hypergroup if $M(X)$ is an associative algebra with respect to a convolution $\star$ satisfying the following conditions:

(1) $M_{1}(X) \star M_{1}(X) \subset M_{1}(X)$;

(2) the convolution is separately continuous from $M_{1}(X) \times M_{1}(X)$ in $M_{1}(X)$ with respect to the weak* topology;

(3) the map $\left(r, r^{\prime}\right) \mapsto \delta_{r} \star \delta_{r^{\prime}}$ is continuous from $X \times X$ in $M(X)$ with the weak* topology;

(4) there exists an element $e$ in $X$, necessarily unique, such that $\delta_{e} \star \nu=\nu \star \delta_{e}=\nu$ for every $v$ in $M(X)$;

(5) there exists an involutive homomorphism from $X$ onto $X, r \mapsto \breve{r}$, which extends naturally to $M(X)$ and satisfies $(\mu \star \nu)=\check{\nu} \star \check{\mu}$, for every $\mu$ and $\nu$ in $M(X)$.

(6) $r=r^{\prime}$ if and only if $e \in \operatorname{supp}\left(\delta_{r^{\prime}} \star \delta_{\check{r}}\right)$;

(7) for every compact set $K \subset X$ and for every neighborhood $V$ of $K$, there exists a neighborhood $U$ of $e$ such that

(a) if $\operatorname{supp}(\mu) \subset K$ and $\operatorname{supp}(\nu) \subset U$, then $\operatorname{supp}(\mu \star v), \operatorname{supp}(\nu \star \mu) \subset V$;

(b) if $\operatorname{supp}(\mu) \subset K$ and $\operatorname{supp}(\nu) \subset V^{c}$, then the supports of $\mu \star \check{v}$ and $\check{\mu} \star \nu$ are disjoint from $U$.

A character on a commutative hypergroup $X$ is a nontrivial, continuous, bounded function $\chi: X \mapsto \mathbb{C}$ such that $\left(\chi, \delta_{r} \star \delta_{r^{\prime}}\right)=\chi(r) \chi\left(r^{\prime}\right)$, for every $r, r^{\prime}$ in $X$. If $\chi(\check{r})=\overline{\chi(r)}$, for every $r$ in $X$, then $\chi$ is an hermitian character. 
Harmonic NA groups Let $\mathfrak{n}$ be a two-step real nilpotent Lie algebra, with an inner product $\langle\cdot, \cdot\rangle_{\mathfrak{n}}$. Write $\mathfrak{n}$ as an orthogonal sum $\mathfrak{n}=\mathfrak{v} \oplus \mathfrak{z}$, where $\mathfrak{z}$ is the center of $\mathfrak{n}$. For each $Z$ in $\mathfrak{z}$, define the map $J_{Z}: \mathfrak{v} \rightarrow \mathfrak{v}$ by the formula

$$
\left\langle J_{Z} X, X^{\prime}\right\rangle_{\mathfrak{n}}=\left\langle\left[X, X^{\prime}\right], Z\right\rangle_{\mathfrak{n}} \quad \forall X, X^{\prime} \in \mathfrak{v} .
$$

We say that the Lie algebra $\mathfrak{n}$ is $H$-type if

$$
J_{Z}^{2}=-|Z|^{2} I_{\mathfrak{v}} \quad \forall Z \in \mathfrak{z},
$$

where $I_{\mathfrak{v}}$ is the identity on $\mathfrak{v}$. The dimension of $\mathfrak{v}$ is even, $\operatorname{dim} \mathfrak{v}=2 \mathrm{~m}$, say; we denote the dimension of the center $z$ by $k$. A connected and simply connected Lie group $N$ whose Lie algebra is an $H$-type algebra is said to be an $H$-type group.

Let $N A$ be the semidirect product of the Lie groups $N$ and $A=\mathbb{R}^{+}$with respect to the action of $A$ on $N$ given by the dilations $(X, Z) \mapsto\left(a^{1 / 2} X, a Z\right)$. As customary we write $(X, Z, a)$ for the element $n a=\exp (X+Z) a$, where $X$ is in $\mathfrak{v}, Z$ in $\mathfrak{z}$ and $a$ in $A$. It can easily be checked that the product law in $N A$ is given by

$$
(X, Z, a)\left(X^{\prime}, Z^{\prime}, a^{\prime}\right)=\left(X+a^{1 / 2} X^{\prime}, Z+a Z^{\prime}+\frac{1}{2} a^{1 / 2}\left[X, X^{\prime}\right], a a^{\prime}\right) .
$$

The inverse of $(X, Z, a)$ is $\left(-a^{-1 / 2} X,-a^{-1} Z, a^{-1}\right)$. We denote by $Q=m+k$ the homogeneous dimension of $N$. A left Haar measure on $N A$ is given by

$$
d x=a^{-Q-1} d X d Z d a,
$$

where $d X, d Z$ and $d a$ are Lebesgue measures on $\mathfrak{n}, \mathfrak{z}$ and $\mathbb{R}^{+}$respectively. We endow $N A$ with the left-invariant Riemannian structure induced by the following inner product on the Lie algebra $n \oplus \mathbb{R}$ of $N A$

$$
\left\langle(X, Z, \alpha),\left(X^{\prime}, Z^{\prime}, \alpha^{\prime}\right)\right\rangle=\left\langle(X, Z),\left(X^{\prime} Z^{\prime}\right)\right\rangle+\alpha \alpha^{\prime} \quad X \in \mathfrak{v}, Z \in \mathfrak{z}, \alpha \in \mathbb{R} .
$$

The expression of the volume density in geodesic polar coordinates is $[1,7]$

$$
\Lambda(\rho)=2^{2 Q}\left(\sinh \frac{\rho}{2}\right)^{2 m+k}\left(\cosh \frac{\rho}{2}\right)^{k} d \rho .
$$

The fact that $\Lambda$ depends only on the geodesic distance $\rho$ from the identity implies that, as a Riemannian manifold, $N A$ is a harmonic space [7]. Notice that once we have an $H$-type algebra $\mathfrak{n}$, the construction of the associated $N A$ group is straightforward.

We say that a function $F: N A \rightarrow \mathbb{C}$ is radial if $F(x)$ depends only on the geodesic distance $|x|$ of the point $x$ from the identity $o=(0,0,1)$ in $N A$. 
Given a locally integrable function $F$ on $N A$ we denote by $\mathscr{R} F$ the radial function on $N A$ given by

$$
\mathscr{R} F(x)=\int_{S_{r}} F(y) d \sigma_{r}(y) \quad x \in N A, r=|x|,
$$

where $S_{r}=\{x \in N A:|x|=r\}$ is the geodesic sphere of radius $r$ centered at the identity and $\sigma_{r}$ is the surface measure supported on $S_{r}$ normalized so that $\int_{S_{r}} d \sigma_{r}=1$. Given a measure $\mu$ in $M(N A)$ we denote by $\mathscr{R} \mu$ the measure defined by the equality $(\mathscr{R} F, \mu)=(F, \mathscr{R} \mu)$, for every $F$ in $C_{0}(N A)$. We say that a measure $\mu$ in $M(N A)$ is radial if $\mathscr{R} \mu=\mu$.

\section{Hypergroups associated to $N A$ groups}

Let $X$ be the locally compact space given by the half line $[0,+\infty)$ endowed with the Euclidean topology. If $f$ is in $C_{0}(X)$ denote by $F$ the radial function on $N A$ defined by $F(x)=f(|x|)$ for every $x$ in $N A$. For $r$ and $r^{\prime}$ in $X$ let $\delta_{r} \star \delta_{r^{\prime}}$ be the measure on $X$ defined by the equality

$$
\left(f, \delta_{r} \star \delta_{r^{\prime}}\right)=\left(F, \sigma_{r} * \sigma_{r}\right)=\int_{S_{r^{\prime}}} \int_{S_{r}} F(x y) d \sigma_{r}(x) d \sigma_{r^{\prime}}(y) \quad \forall f \in C_{0}(X) .
$$

The convolution $\star$ naturally extends to $M(X)$.

THEOREM 2.1. The locally compact space $X$ is a hypergroup with respect to the convolution $\star$ defined by (2) and the involution $r \mapsto \check{r}=r$ on $X$.

PROOF. The proof is a verification of conditions (1)-(7) defining a hypergroup. A detailed proof is given in [3].

Therefore we can associate a hypergroup to every $N A$ group. Our next aim is to write the explicit expression for the convolution $\star$ defined by (2).

It is easy to see that $\Lambda(u) d u$ is a translation-invariant positive Radon measure on the hypergroup $X$.

For any complex number $\lambda$ denote by $\Phi_{\lambda}: N A \rightarrow \mathbb{C}$ the spherical function on $N A$ associated to the eigenvalue $-\left(\lambda^{2}+Q^{2} / 4\right)$ of the Laplace-Beltrami operator on $N A$. The spherical function $\Phi_{\lambda}$ is bounded if and only if $|\operatorname{Im} \lambda| \leq Q / 2$ [7]. Denote

$$
\varphi_{\lambda}(r)=\Phi_{\lambda}(x) \text { if }|x|=r .
$$

It is easy to see that the set of the hermitian characters of $X$ is $\left\{\varphi_{\lambda}: \lambda \in \mathbb{R} U\right.$ $[-i Q / 2, i Q / 2]\}$. 
The spherical functions $\left\{\Phi_{\lambda}\right\}_{\lambda \in C}$ on $N A$ can be expressed in terms of hypergeometric functions. More precisely we have $([1,2])$

$$
\varphi_{\lambda}(r)={ }_{2} F_{1}\left(Q / 2-i \lambda, Q / 2+i \lambda ;(2 m+k+1) / 2 ;-\sinh ^{2}(r / 2)\right),
$$

where ${ }_{2} F_{1}$ denotes the hypergeometric function according to the notation in [8].

Flensted-Jensen and Koornwinder proved the following equality [9, formulae (4.2) and (4.17)]

$$
\varphi_{\lambda}(r) \varphi_{\lambda}(t)=\int_{0}^{+\infty} \varphi_{\lambda}(u) T(r, t, u) \Lambda(u) d u \quad \forall r, t \in[0,+\infty),
$$

where for $u \notin(|r-t|,|r+t|)$

$$
T(r, t, u)=0
$$

and for $|r-t|<u<|r+t|$

$$
\begin{aligned}
T(r, t, u)= & \frac{\Gamma((2 m+k+1) / 2)}{2^{2 Q} \sqrt{\pi} \Gamma(m) \Gamma(k / 2)}(\sinh (r / 2) \sinh (t / 2) \sinh (u / 2))^{-2 m-k+1} \\
& \times \int_{0}^{\pi}\left(g_{(r, t, u)}(\theta)\right)_{+}^{m-1}(\sin \theta)^{k-1} d \theta
\end{aligned}
$$

with $g_{(r, t, u)}(\theta)=1-\left(\cosh \frac{r}{2}\right)^{2}-\left(\cosh \frac{t}{2}\right)^{2}-\left(\cosh \frac{u}{2}\right)^{2}+2 \cosh \frac{r}{2} \cosh \frac{t}{2} \cosh \frac{u}{2} \cos \theta$. Here $(z)_{+}=z$ for $z>0$ and $(z)_{+}=0$ for $z \leq 0$.

THEOREM 2.2. For every $r$ and $t$ in $X=[0,+\infty)$, we have

$$
d\left(\delta_{r} \star \delta_{r^{\prime}}\right)(u)=T(r, t, u) d \eta(u) \quad \forall u \in X
$$

PROOF. Let $r$ and $t$ be in $X$ and let $x$ and $y$ be in NA such that $|x|=r$ and $|y|=t$. In [7] the following relation is proved

$$
\mathscr{R}\left({ }_{x} \Phi_{\lambda}\right)(y)=\Phi_{\lambda}(x) \Phi_{\lambda}(y)=\varphi_{\lambda}(r) \varphi_{\lambda}(t) \quad \forall \lambda \in \mathbb{C},
$$

where ${ }_{x} \Phi_{\lambda}(y):=\Phi_{\lambda}(x y)$. For $|\operatorname{Im} \lambda| \leq Q / 2$, denote by $\widehat{F}(\lambda)=\int_{N A} F(x) \Phi_{\lambda}(x) d x$ the spherical transform of an integrable radial function $F$ on $N A$.

Let $f$ be a $C^{\infty}$ function with compact support on $X$ and define $F: N A \rightarrow \mathbb{C}$ by $F(x)=f(|x|)$, for every $x$ in $N A$. By the inversion formula for the spherical 
transform on $N A[10]$ and by the Fubini Theorem we have

$$
\begin{aligned}
\left(f, \delta_{r} \star \delta_{r^{\prime}}\right) & =\int_{S_{r}} \int_{S_{t}} F(x y) d \sigma_{r}(x) d \sigma_{t}(y) \\
& =\int_{S_{r}} \int_{S_{t}} c \int_{-\infty}^{+\infty} \widehat{F}(\lambda) \Phi_{\lambda}(x y)|\mathbf{c}(\lambda)|^{-2} d \lambda d \sigma_{r}(x) d \sigma_{t}(y) \\
& =c \int_{-\infty}^{+\infty} \int_{S_{r}} \mathscr{R}\left({ }_{x} \Phi_{\lambda}\right)(y) d \sigma_{r}(x) \widehat{F}(\lambda)|\mathbf{c}(\lambda)|^{-2} d \lambda \\
& =c \int_{-\infty}^{+\infty} \varphi_{\lambda}(r) \varphi_{\lambda}(t) \widehat{F}(\lambda)|\mathbf{c}(\lambda)|^{-2} d \lambda \\
& =c \int_{-\infty}^{+\infty} \int_{0}^{+\infty} \varphi_{\lambda}(u) T(r, t, u) \Lambda(u) d u \widehat{F}(\lambda)|\mathbf{c}(\lambda)|^{-2} d \lambda \\
& =\int_{0}^{+\infty} f(u) T(r, t, u) \Lambda(u) d u,
\end{aligned}
$$

where $c$ is a constant depending only on $m$ and $k$ and

$$
\mathbf{c}(\lambda)=\frac{2^{Q-2 i \lambda} \Gamma(2 i \lambda) \Gamma((2 m+k+1) / 2)}{\Gamma(Q / 2+i \lambda) \Gamma((m+1) / 2+i \lambda)} .
$$

It is known that if $\mathfrak{n}=\mathfrak{v} \oplus \mathfrak{z}$ and $\mathfrak{n}^{\prime}=\mathfrak{v}^{\prime} \oplus \mathfrak{z}^{\prime}$ are $H$-type algebra such that $\operatorname{dim} \mathfrak{n}=\operatorname{dim} \mathfrak{n}^{\prime}$ and $\operatorname{dim} \mathfrak{z}=\operatorname{dim} \mathfrak{z}^{\prime}$ then $\mathfrak{n}$ and $\mathfrak{n}^{\prime}$ are not necessarily isomorphic (see [5]); however, the associated hypergroups are isomorphic.

THEOREM 2.3. If $\mathfrak{n}=\mathfrak{v} \oplus \mathfrak{z}$ and $\mathfrak{n}^{\prime}=\mathfrak{v}^{\prime} \oplus \mathfrak{z}^{\prime}$ are two H-type algebras such that $\operatorname{dim} \mathfrak{n}=\operatorname{dim} \mathfrak{n}^{\prime}$ and $\operatorname{dim} \mathfrak{z}=\operatorname{dim} \mathfrak{z}^{\prime}$, then the hypergroups associated to $\exp (\mathfrak{n}+\mathbb{R})$ and $\exp \left(\mathfrak{n}^{\prime}+\mathbb{R}\right)$ are isomorphic.

PROOF. Although the result follows by Theorem 2.2, we give here a different proof which does not involve formula (3). From (2) and from the expression of the product law in NA it is clear that the result follows if we prove that the convolution of radial functions on $N A$ does not depend on the bracket of the Lie algebra $n$. Fix a point $\left(X^{\prime \prime}, Z^{\prime \prime}, a^{\prime \prime}\right)$ in $N A$ and let $\left(0, Z^{\prime}, a^{\prime}\right)$ be in $N A$ such that $\left|\left(X^{\prime \prime}, Z^{\prime \prime}, a^{\prime \prime}\right)\right|=\left|\left(0, Z^{\prime} a^{\prime}\right)\right|$. Let $F$ and $\Psi$ be radial functions on $N A$. Since $F * \Psi$ is a radial function on $N A$, we have

$$
\begin{aligned}
(F * \Psi) & \left(X^{\prime \prime}, Z^{\prime \prime}, a^{\prime \prime}\right) \\
& =(F * \Psi)\left(0, Z^{\prime}, a^{\prime}\right) \\
& =\int_{N A} F(X, Z, a) \Psi\left((X, Z, a)^{-1}\left(0, Z^{\prime}, a^{\prime}\right)\right) a^{-Q-1} d X d Z d a \\
& =\int_{N A} F(X, Z, a) \Psi\left(-a^{-1 / 2} X, a^{-1}\left(Z^{\prime}-Z\right), a^{\prime} a^{-1}\right) a^{-Q-1} d X d Z d a
\end{aligned}
$$


We are done since the last expression does not depend on the Lie bracket of $\mathfrak{n}$.

REMARK. Wildberger [12] noted that there is a hypergroup associated to any Gelfand pair $(G, K)$. When $N A$ is a noncompact symmetric space $G / K$, the compact Lie group $K$ acts transitively on the geodesic spheres of $G / K$; moreover $(G, K)$ is a Gelfand pair. For a generic $N A$ harmonic space does not exist a group $K$ with these properties (see [6]); however it is possible to generalize the theory of Gelfand pairs to this situation [4]. Let us give more information on this topic.

Damek and Ricci [7] introduced the notion of an averaging projection $\mathscr{R}$ on a Lie group $S$, which is an idempotent operator on the space of $C^{\infty}$ functions with compact support on $S$ satisfying suitable conditions. The functions in the image of $\mathscr{R}$ are called radial. The convolution of radial functions is a radial function and the space $L_{\mathscr{R}}^{1}(S)$ of radial integrable functions is a Banach algebra. If the convolution algebra $L_{\mathscr{R}}^{1}(S)$ is commutative it plays the same role played by the Banach algebra $L^{1}(K \backslash G / K)$ in the theory of Gelfand pairs [4]. Damek and Ricci [7] proved that the operator $\mathscr{R}$ defined by (1) is an averaging projection on $N A$ and that the Banach algebra $L_{\mathscr{R}}^{1}(N A)$ is commutative.

This point of view is not emphasized in this paper, however we think that it could be interesting to study connections between hypergroups and Lie groups admitting an averaging projection.

\section{Acknowledgements}

Theorem 2.1 and Theorem 2.3 are contained in the Ph.D. Thesis [3] of the author, who is grateful to her advisor Fulvio Ricci for his precious help and encouragement. The author also wishes to thank Jean-Philippe Anker and Norman Wildberger for many useful conversations.

\section{References}

[1] J.-Ph. Anker, E. Damek and C. Yacoub, 'Spherical analysis on harmonic AN groups', Ann. Scuola Norm. Sup. Pisa Cl. Sci. (4) 23 (1996), 643-679.

[2] F. Astengo, 'A class of $L^{p}$ convolutors on harmonic extensions of $H$-type groups', J. Lie Theory 5 (1995), 147-164.

[3] B. Di Blasio, Analisi di Fourier sferica su estensioni risolubili di gruppi di Heisenberg generalizzati (Ph.D. Thesis, Politecnico di Torino, 1997).

[4] _ , 'An extension of the theory of Gelfand pairs to radial functions on Lie groups', Boll. Un. Mat. Ital. B (7) 11 (1997), 623-642.

[5] M. Cowling, A. H. Dooley, A. Korányi and F. Ricci, ' $H$-type groups and Iwasawa decompositions', Adv. Math. 87 (1991), 1-41. 
[6] E. Damek, 'The geometry of a semidirect extension of a Heisenberg type nilpotent group', Colloq. Math. 53 (1987), 255-268.

[7] E. Damek and F. Ricci, 'Harmonic analysis on solvable extensions of $H$-type groups', J. Geom. Anal. 2 (1992), 213-248.

[8] A. Erdelyi, W. Magnus, F. Oberhettinger and G. Tricomi, Higher transcendental functions, Vol. I (McGraw-Hill, New York, 1953).

[9] M. Flensted-Jensen and T. Koornwinder, 'The convolution structure for Jacobi function expansions', Ark. Mat. 11 (1973), 245-262.

[10] F. Ricci, 'The spherical transform on harmonic extensions of $\boldsymbol{H}$-type groups', Rend. Sem. Mat. Univ. Politec. Torino 50 (1992), 381-392.

[11] R. Spector, 'Aperçu de la théorie des hypergroupes', in: Analyse harmonique sur les groupes de Lie (Sém. Nancy-Strasbourg, 1973-75) Lecture Notes in Math. 497 (Springer, Berlin, 1975) pp. 643-673.

[12] N. J. Wildberger, 'Hypergroups, symmetric spaces, and wrapping maps', in: Probability measures on groups and related structures, XI, (Oberwolfach, 1994) (World Sci. Publishing, River Edge, NJ, 1995) pp. 406-425.

Dipartimento di Matematica

Università degli studi di Roma 'Tor Vergata'

via della Ricerca scientifica 1

00133 Roma

Italy

e-mail: diblasio@axp.mat.uniroma2.it 